\title{
Type 1 and type 2 diabetes mellitus: are they mutually exclusive?
}

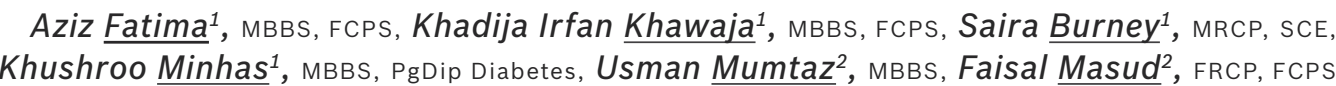

INTRODUCTION With advancement in the understanding of the pathogenesis underlying diabetes mellitus (DM), the boundary between type 1 and type 2 DM (T1DM and T2DM) does not seem to be as clear cut as previously thought. This study was designed to test the possibility of overlap between the spectra of immune-mediated $\mathrm{DM}$ and insulin resistance.

METHODS To test for the possibility of overlap, we looked for autoantibodies typical of T1DM in patients with classical T2DM, and insulin resistance in patients with T1DM. Autoantibodies against islet cell antigen, glutamic acid decarboxylase-65 and insulinoma-associated antigen-2 were tested in 82 patients with T2DM and 27 patients with T1DM. The patients had been diagnosed on clinical criteria using standard laboratory techniques. Clinical parameters of diagnostic importance were noted, and homeostatic model assessment of insulin resistance (HOMA-IR) was calculated using fasting insulin and fasting blood glucose ratio.

RESULTS Autoantibodies against one or more beta cell antigens were detected in $12.19 \%$ of patients clinically diagnosed to have T2DM, and insulin resistance (HOMA-IR > 2.5) was diagnosed in 37.03\% of patients with T1DM. It was not possible to identify any combination of clinical or biochemical markers that could predict autoantibody positivity in T2DM patients. T1DM patients with insulin resistance had a significantly higher body mass index than their insulin-sensitive counterparts $(p=0.02)$.

CONCLUSION Autoantibodies against beta cell antigens are detectable in insulin-resistant T2DM patients, and insulin resistance may be present in relatively overweight T1DM patients. No differentiating clinical features that might predict autoantibody positivity in T2DM patients were found.

Keywords: autoantibodies, insulin resistance, type 1 diabetes mellitus, type 2 diabetes mellitus

\section{INTRODUCTION}

Diabetes mellitus (DM) is classically divided into two distinct categories - type 1 and type 2 (T1DM and T2DM), which account for the majority of cases. T1DM is characterised by beta cell destruction that usually occurs in the early stages of life. This beta cell destruction is thought to be mediated by an immune response to triggering factors that are not yet clearly defined. It often results in the rapid onset of an insulin-deficient state and consequent hyperglycaemia, which is clinically evidenced by weight loss, polyuria, and sometimes, diabetic ketoacidosis. While the incidence of T1DM is higher in children and adolescents, it can occur at any age. (1) The presence of serum markers of autoimmune beta cell destruction, such as autoantibodies against islet cell antigen (ICA), glutamic acid decarboxylase-65 (GAD-65) and insulinoma-associated antigen-2 (IA2) support a diagnosis of T1DM. conversely T2DM is characteristically linked to insulin resistance, with relative preservation of the beta cells at onset. This results in compensatory overproduction of insulin, leading to a state of hyperinsulinaemia, which can be evidenced by clinical markers such as centripetal obesity, acanthosis nigricans and the presence of a fatty liver. Although T2DM typically occurs after the age of 30 years, patients are presenting earlier due to the obesity epidemic in adolescents and young adults. HOMA-IR is a convenient method for estimating insulin resistance and has been validated against the hyperinsulinaemic euglycaemic clamp, which is both labour intensive and clinically invasive. ${ }^{(2)}$

The conventional demarcation of DM into two distinct clinical types is challenged by patients whose phenotype does not fit into either category, even after uncommon DM types such as maturity onset diabetes of the young, latent autoimmune diabetes of adults (LADA) and rare endocrinopathies have been ruled out. Due to the existence of these overlap cases, the traditional classification of DM is now questioned, and the two types of DM are proposed to be merely two ends of a continuous spectrum. ${ }^{(3)}$ There are reports of insulin resistance in patients who have apparent T1DM, ${ }^{(4,5)}$ and classical type 1 autoantibodies have been detected in obese patients who phenotypically have T2DM.(6,7) These reports have led us to hypothesise that there is an overlap in the spectra of the two classical types of DM. To test this, we measured the

${ }^{1}$ Endocrinology Unit and Diabetes Management Centre, ${ }^{2}$ Department of Medicine, Unit 4, Services Institute of Medical Sciences and Services Hospital, Lahore, Pakistan

Correspondence: Dr Faisal Masud, Principal and Professor of Medicine, 801-C, Akbar Chowk, Faisal Town, Lahore, Pakistan. dr.faisalmasud@gmail.com 
markers of autoimmune beta cell damage in both patients with T1DM and those with T2DM.

\section{METHODS}

This cross-sectional study was conducted in the Endocrinology Unit and Diabetes Management Centre of Services Hospital, a tertiary care hospital in Lahore, Pakistan. The study was approved by the Institutional Review Board of Services Hospital/Services Institute of Medical Sciences. All research procedures were conducted in accordance with the principles laid down in the Helsinki Declaration. A total of 183 diabetic patients visiting the outpatient diabetes clinic were screened for inclusion through semipurposive sampling. Patients without a clear-cut T1DM or T2DM diagnosis were excluded from the study. LADA was not a definite diagnosis in our patient pool because routine autoantibody testing was not available; however, it was excluded on the basis of referenced clinical criteria. ${ }^{(8)}$ Patients who had poor metabolic control and concomitant conditions, or were on drugs affecting insulin sensitivity/autoantibody detection were also excluded from the study. A total of 125 patients with either T1DM or T2DM were recruited. These patients went through the run-in phase of the study following the procurement of free, written informed consent. The clinical diagnosis of either type of DM was made by three endocrinologists who worked independently. The main criteria used for differentiating between T1DM and T2DM are shown in Table I. Of the 125 patients, 11 were subsequently excluded, mainly due to poor glycaemic control, and/or the lack of follow-up on the scheduled visit date. The remaining 114 patients were sampled for autoantibodies, fasting insulin level and fasting glucose level on their final visit. As the serum samples from five patients could not be processed, the final sample size of our study was 109 patients, which comprised 82 patients with T2DM and 27 with T1DM.

During the run-in phase, all long-acting antidiabetic drugs that were likely to affect insulin sensitivity were withdrawn for a duration equal to five half-lives of the medicine. If required, the patients were switched to short-acting insulin or repaglinide until one day before the sampling. Patients were instructed to monitor their blood glucose levels at home and report back if the level exceeded $250 \mathrm{mg} / \mathrm{dL}$ at any time during the run-in phase. If this occurred, the patient was excluded from the study and his/her original medicines were reintroduced. Thereafter, all patients were called for a second visit, prior to which they had to fast overnight. After a detailed history was taken and a thorough examination done, $6 \mathrm{~mL}$ of venous blood was drawn for autoantibody assays and measurement of insulin and glucose levels. Blood samples were allowed to clot for half an hour, after which the samples were centrifuged at $2,500 \mathrm{rpm}$ for $15 \mathrm{mins}$ at a temperature of $4^{\circ} \mathrm{C}$. The separated serum was collected and stored in duplicate microcentrifuge tubes at $-20^{\circ} \mathrm{C}$ until processing.
Table I. Clinical criteria used for the classification of diabetes mellitus.

\begin{tabular}{ll}
\hline Type 1 & Type 2 \\
\hline - Onset < 30 years of age & - Onset > 30 years of age \\
- Rapid onset & - Insidious onset \\
- Marked osmotic symptoms & - Family history of T2DM \\
- History of significant & - Overweight/obese \\
weight loss & - WC $\geq 80 \mathrm{~cm}$ for females; \\
- Documented history of & WC $\geq 90 \mathrm{~cm}$ for males \\
diabetic ketoacidosis & - Positive acanthosis \\
- Absence of acanthosis & nigricans* \\
nigricans* & - Presence of fatty liver** \\
- Absence of fatty liver** & - Lack of insulin requirement \\
- Insulin requirement & $\begin{array}{l}{ }^{*} \text { Acanthosis nigricans was checked at the nape of the neck. **Diagnosis of } \\
\text { fatty liver was made if a clinically enlarged liver was found and confirmed using } \\
\text { ultrasonography. } \\
\text { T2DM: type 2 diabetes mellitus; WC: waist circumference }\end{array}$
\end{tabular}

Insulin levels were measured using an insulin enzymelinked immunosorbent assay (ELISA) kit (Nova Tec Immunodiagnostica $\mathrm{GmBH}$, Dietzenbach, Germany; sensitivity limit $2 \mu \mathrm{IU} / \mathrm{mL}$, intra-assay variation $2 \%$; interassay variation $6 \%$ ). Autoantibodies against IA2 in the sera were measured using quantitative enzyme immunoassay (reference kit no. EA105/96; DLD Diagnostika GmBh, Hamburg, Germany). Assay precision had been calculated using two samples at different concentrations, with an intra-assay variation of $1.9 \%-3.5 \%$, and an interassay coefficient of variation of $4.9 \%-9.8 \%$. Values $\geq 7.5 \mathrm{U} / \mathrm{mL}$ were considered positive for IA2 autoantibodies. The assay is reported to have a clinical sensitivity of $66 \%$ and a specificity of $99 \%$. Sera were also assayed for autoantibodies against GAD-65 using ELISA (reference kit no. EA104/96; DLD Diagnostika GmbH, Hamburg, Germany; intra-assay coefficient of variation $7.3 \%-8.5 \%$, interassay variation $5.2 \%-5.7 \%$, clinical sensitivity $92 \%$, specificity $98 \%$ ). The recommended cutoff value for positivity (i.e. $\geq 10 \mathrm{U} / \mathrm{mL}$ ) was used. Autoantibodies to ICA were detected using enzyme immunoassay (Medizym, reference kit no. 3804; Medipan $\mathrm{GmbH}$, Berlin, Germany; intra- and interassay coefficient of variation of $2.5 \%-3.3 \%$ and $3.3 \%-4.8 \%$, respectively). Values $\geq 1.0$ binding index were considered positive. Insulin resistance was estimated using HOMA-IR, which was calculated using the following formula: fasting glucose $(\mathrm{mmol} / \mathrm{L}) \times$ fasting insulin $(\mu \mathrm{U} / \mathrm{ml}) / 22.5$. Patients with an HOMA-IR value $>2.5$ were deemed to have insulin resistance. ${ }^{(10)}$

\section{RESULTS}

The final study population consisted of 109 patients - 82 were diagnosed with T2DM (46 women, 36 men) and 27 patients with T1DM (16 women, 11 men). Descriptive statistics for continuous variables are shown in Table II.

One or more autoantibodies were detected in 29 (26.6\%) of the 109 patients studied. Among the 27 patients with T1DM, 
$19(70.4 \%)$ were positive for at least one autoantibody, $12(44.4 \%)$ were positive for two autoantibodies, while all three autoantibodies were detected in $4(14.8 \%)$. ICA and GAD-65 autoantibodies showed a similar frequency in patients with T1DM - 16 were positive for ICA, while 15 were positive for GAD-65. IA2 was detected in eight patients with T1DM.

Of the 82 patients with T2DM, 10 (12.2\%) tested positive for at least one autoantibody, 1 (1.2\%) showed dual autoantibody positivity, while all three autoantibodies were present in $2(2.4 \%)$. IA2 was the most common autoantibody detected in patients with T2DM, with a frequency of $7 / 82(8.5 \%)$, followed by GAD-65 (5/82, 6.1\%). Only 3 (3.6\%) patients with T2DM tested positive for ICA autoantibodies. Fig. 1 shows a comparison between the clinical characteristics of patients with T2DM with positive and negative autoantibody status. No significant differences were seen between the two groups for all the variables studied, which included age at diagnosis $(p=1.0)$, BMI $(p=0.9)$, waist circumference $(p=0.7)$, area of acanthosis nigricans $(p=0.4)$, enlarged fatty liver $(p=0.5)$, HOMA-IR value $(p=0.5)$, and presence of insulin resistance $(p=1.0)$.

$78 \%$ of the patients with T2DM and $37 \%$ of those with T1DM had insulin resistance (defined as HOMA-IR > 2.5). $59(71.9 \%)$ of the patients with T2DM and 7 (5.9\%) of those with T1DM showed clinical evidence of an enlarged fatty liver, a marker of insulin resistance. Comparison of the clinical features of T1DM patients with and without insulin resistance showed that the two groups were similar in terms of age at diagnosis $(p=0.5)$, waist circumference $(p=0.1)$ and degree of acanthosis nigricans $(p=0.8)$. However, patients with T1DM who have HOMA-IR $>2.5$ had a significantly higher BMI (mean \pm standard deviation [SD]: $25.1 \pm 7.4$, standard error of the mean [SEM] 2.3) than insulin-sensitive patients with T1DM (mean \pm SD: $19.4 \pm 4.7$, SEM 1.1) $(p=0.02)$.

\section{DISCUSSION}

While the prevalence of T1DM has risen in the last decade, that of T2DM has reached epidemic proportions. ${ }^{(11)}$ This dramatic rise has been attributed to changing lifestyles, resulting in a rise in obesity and insulin resistance across all strata of society. This epidemic of diabesity, coupled with the decreasing age of onset of T2DM, has resulted in an increasing number of patients in whom the distinction between the two classical DM types has become blurred. With nearly a third of the young population overweight ${ }^{(12)}$ and consequently insulin resistant, one can expect some overlap of this state with autoimmune DM. In the same way an insulin-resistant patient may catch pneumonia or develop arthritis, the patient can also end up 'catching' T1DM because the presence of obesity or genetic insulin resistance does not rule out, or confer protection against, the development of autoimmune T1DM. Indeed, Suzuki et al have reported cases of autoimmune insulinrequiring DM superimposed on classical T2DM, years after the
Table II. Clinical characteristics of patients with type 1 and type 2 diabetes mellitus.

\begin{tabular}{lccc}
\hline \multirow{2}{*}{$\begin{array}{l}\text { Clinical } \\
\text { characteristics }\end{array}$} & \multicolumn{2}{c}{ Mean \pm SD } & p-value \\
\cline { 2 - 3 } & $\begin{array}{c}\text { Type } \mathbf{1} \\
(\mathbf{n}=\mathbf{2 7})\end{array}$ & $\begin{array}{c}\text { Type 2 } \\
(\mathbf{n}=\mathbf{8 2})\end{array}$ & \\
\hline DM onset age $(\mathbf{y r s})$ & $17.48 \pm 9.87$ & $42.22 \pm 8.80$ & 0.000 \\
$\mathbf{B M I}\left(\mathbf{k g} / \mathbf{m}^{\mathbf{2}}\right)$ & $21.50 \pm 6.34$ & $28.25 \pm 5.55$ & 0.000 \\
$\mathbf{W C}^{*}(\mathbf{c m})$ & $77.11 \pm 15.50$ & $99.90 \pm 10.85$ & 0.000 \\
WHR $^{+}$ & $0.89 \pm 0.06$ & $0.98 \pm 0.12$ & 0.000 \\
Area of AN* $\left.\mathbf{( c m}^{\mathbf{2}}\right)$ & $2.59 \pm 6.71$ & $9.73 \pm 11.86$ & 0.004 \\
HOMA-IR & $4.65 \pm 6.66$ & $5.67 \pm 5.57$ & 0.434 \\
\hline
\end{tabular}

${ }^{*}$ Measured at the level of the iliac crest using a horizontal tape measure. †The widest horizontal measure around the hips was measured as the hip circumference. ${ }^{\ddagger}$ Acanthosis nigricans area was measured at the nape of the neck.

AN: acanthosis nigricans; BMI: body mass index; DM: diabetes mellitus; HOMA-IR: homeostatic model assessment of insulin resistance; SD: standard deviation; WC: waist circumference; WHR: waist-hip ratio

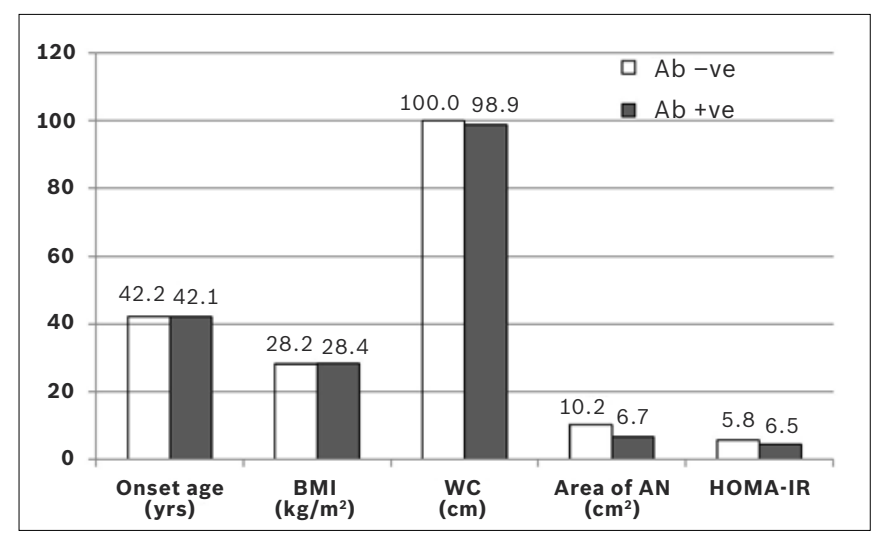

Fig. 1 Comparison between autoantibody-positive $(A b+v e)$ and autoantibody-negative $(A b-v e)$ patients with type 2 diabetes mellitus. Independent samples $t$-test showed that there was no significant difference for all the parameters studied. Data show the absolute mean values.

AN: acanthosis nigricans; BMI: body mass index; HOMA-IR: homeostatic model assessment of insulin resistance; WC: waist circumference

original diagnosis. ${ }^{(13)}$ This would result in the concurrence of autoimmune DM and T2DM in the same patient. In the present study, we tested this hypothesis by measuring autoantibodies against beta cell antigens in patients with T2DM. A control group of patients with T1DM were also tested for comparison purposes.

There were clear and significant differences in the age of onset, BMI, waist circumference, area of acanthosis nigricans, and frequency of fatty liver among the T1DM and T2DM cohorts. This was expected since these criteria were among those used to assign a clinical type to the study subjects, and only clear-cut cases of either type were recruited (patients with indeterminate phenotypes and those in whom a consensus could not be reached were excluded). Although insulin resistance was more prevalent in patients with T2DM, interestingly, $37 \%$ of the patients with T1DM also had an HOMA-IR greater than the cutoff value of 2.5, indicating that insulin resistance could be found not only in patients with T2DM but also in classic cases of T1DM. This finding is particularly significant because the HOMA model tends 
to underestimate insulin resistance in patients with T1DM who have beta cell dysfunction. ${ }^{(14)}$ Multiple studies have also documented insulin resistance among patients with T1DM. ${ }^{(15,16)}$ This trend may reflect the response to environmental influences that promote obesity, which patients with T1DM are similarly exposed to. ${ }^{(15)}$ As postulated by the accelerator hypothesis, rising obesity and insulin resistance are the major factors contributing to the increasing incidence of both T2DM and autoimmune T1DM. ${ }^{(17)}$ In our study, patients with T1DM who were insulin resistant $(37 \%)$, though not overtly obese, still showed a significantly higher BMI than those who had normal insulin sensitivity $(p=0.02)$. This finding seems to support the theory that the boundary between the two types of DM is now blurred. It also emphasises the need for lifestyle intervention that aims for weight control in patients with T1DM.

Islet autoantibodies were detected in $70.3 \%$ of the T1DM cohort, with the majority testing positive for multiple autoantibodies. This is in accordance with the reported literature. ${ }^{(18,19)}$ Of greater interest is the finding that $12.2 \%$ of the T2DM cohort also tested positive for at least one autoantibody against islet cells, including three patients with multiple autoantibody positivity. This finding favours our hypothesis that autoimmune markers are detectable in phenotypically insulinresistant patients with T2DM. Although GAD-65 is reported in the literature as being the most frequent autoantibody found among adult diabetics, ${ }^{(20)}$ IA2 was the most common autoantibody found in our T2DM sample. This may be because we did not include patients with LADA in our study, as GAD65 positivity is classically associated with the specific diagnosis of LADA, which is distinct from T2DM. ${ }^{(21)}$ In comparison, Bottazzo et al reported the presence of IA2 to be "infrequent (2.2\%) among type 2 diabetics, but highly predictive of future insulin requirement". ${ }^{(22)}$ Among the three autoantibodies measured in the present study, ICA showed maximum predilection for T1DM, and was the least frequent among the T2DM cohort. Studies across the world have documented ICA as being the most prevalent autoimmune marker of T1DM, with a sensitivity of $70 \%-80 \%$ and a specificity of more than $99 \%$. $^{(23)}$

We found no significant historical, phenotypic or laboratory parameters that predicted autoantibody positivity in patients clinically labelled as T2DM. The mean age of onset, BMI, waist circumference, area of acanthosis nigricans, and even HOMA-IR value of patients with T2DM who showed autoantibody positivity were statistically similar to patients with T2DM who were autoantibody-negative. Other studies have also failed to detect clinical or biochemical features that are predictive of autoimmunity in patients who have apparent T2DM. ${ }^{(24,25)}$ Therefore, the identification of autoantibodypositive adults remains difficult as autoantibody screening of all diabetics is not economically feasible. The presence of autoimmune markers in patients with T2DM may be an incidental finding, as autoantibodies are found in up to $3 \%$ of the nondiabetic population, ${ }^{(19)}$ or it may predict future insulin requirement in affected individuals. ${ }^{(26,27)}$ Complications, and perhaps complete beta cell failure, may be prevented by identification and careful follow-up of these patients with apparent T2DM by clinicians, and prompt institution of insulin therapy. ${ }^{(28,29)}$ While the results of our study have important implications on the modification of future management strategies of diabetes subgroups, these results need to be confirmed using larger, controlled prospective trials.

\section{ACKNOWLEDGEMENTS}

The authors thank SIMS statistical officer, Mr Muhammad Ghias, and laboratory personnel, Mr Muhammad Aslam, for their valuable help in this study.

\section{REFERENCES}

1. American Diabetes Association. Diagnosis and classification of diabetes mellitus. Diabetes Care 2012; 35 Suppl 1:64-71.

2. Matthews DR, Hosker JP, Rudenski AS, et al. Homeostasis model assessment: insulin resistance and beta-cell function from fasting plasma glucose and insulin concentrations in man. Diabetologia 1985; 28:412-9.

3. Brooks-Worrell B, Palmer JP. Is diabetes mellitus a continuous spectrum? Clin Chem 2011; 57:158-61.

4. Tsui H, Paltser G, Chan Y, Dorfman R, Dosch HM. 'Sensing' the link between type 1 and type 2 diabetes. Diabetes Metab Res Rev 2011; 27:913-8.

5. Nadeau KJ, Regensteiner JG, Bauer TA et al. Insulin resistance in adolescents with type 1 diabetes and its relationship to cardiovascular function. J Clin Endocrinol Metab 2010; 95:513-21.

6. Brooks-Worrell B, Palmer JP. Immunology in the Clinic Review Series; focus on metabolic diseases: development of islet autoimmune disease in type 2 diabetes patients: potential sequelae of chronic inflammation. Clin Exp Immunol 2012; 167:40-6.

7. Negishi M, Shimomura K, Proks P, et al. An obese patient with slowly progressive type 1 diabetes diagnosed by ketoacidosis. Intern Med 2010; 49:393-5.

8. Nambam B, Aggarwal S, Jain A. Latent autoimmune diabetes in adults: A distinct but heterogeneous clinical entity. World J Diabetes 2010; 1:111-5.

9. International Diabetes Federation. The IDF consensus worldwide definition of the metabolic syndrome. Brussels: International Diabetes Federation, 2006

10. Friedrich $\mathrm{N}$, Thuesen $\mathrm{B}$, Jørgensen $\mathrm{T}$, et al. The association between IGF-1 and insulin resistance: a general population study in Danish adults. Diabetes Care 2012; 35:768-73.

11. Alberti G, Zimmet P, Shaw J, et al. Type 2 diabetes in the young: the evolving epidemic: the international diabetes federation consensus workshop. Diabetes Care 2004; 27:1798-811.

12. LaFontaine T. Physical Activity: The Epidemic of Obesity and Overweight Among Youth: Trends, Consequences, and Interventions. American Journal of Lifestyle Medicine 2008; 2:30-36.

13. Suzuki T, Takahashi K, Miyamoto S, et al. "Type 1 on type 2 " diabetes mellitus: autoimmune type 1 diabetes superimposed on established type 2 diabetes. Intern Med 2007; 46:1957-62.

14. Wallace TM, Levy JC, Matthews DR. Use and abuse of HOMA modeling. Diabetes Care 2004; 27:1487-95.

15. Szadkowska A, Pietrzak I, Bodalska-Lipińska J, et al. [Insulin resistance in young type 1 diabetic patients]. Diabetologia Doświadczalna i Kliniczna 2006; 6:293-8. Polish.

16. Xu P, Cuthbertson D, Greenbaum C, Palmer JP, Krischer JP; Diabetes Prevention Trial-Type 1 Study Group. Role of insulin resistance in predicting progression to type 1 diabetes. Diabetes Care 2007; 30:2314-20.

17. Pozzilli P, Guglielmi C, Caprio S, Buzzetti R. Obesity, autoimmunity, and double diabetes in youth. Diabetes Care 2011; 34 Suppl 2:166-70. 
18. Verge CF, Gianani R, Kawasaki E, et al. Number of autoantibodies (against insulin, GAD or ICA512/IA2) rather than particular autoantibody specificities determines risk of type 1 diabetes. J Autoimmun 1996; 9:379-83.

19. Winter WE, Harris N, Schatz D. Immunologic markers in the diagnosis and prediction of autoimmune, type $1 \mathrm{~A}$ diabetes. Clinical Diabetes 2002; 20:183-91.

20. Falorni A, Brozzetti A. Diabetes-related antibodies in adult diabetic patients. Best Pract Res Clin Endocrinol Metab 2005; 19:119-33.

21. Zimmet PZ, Tuomi T, Mackay IR. Latent autoimmune diabetes mellitus in adults (LADA): the role of antibodies to glutamic acid decarboxylase in diagnosis and prediction of insulin dependency. Diabet Med 1994; 11:299-303.

22. Bottazzo GF, Bosi E, Cull CA, et al. IA-2 antibody prevalence and risk assessment of early insulin requirement in subjects presenting with type 2 diabetes (UKPDS 71). Diabetologia 2005; 4:703-8.

23. Winter WE, Schatz DA. Autoimmune markers in diabetes. Clin Chem 2011; 57:168-75.

24. Juneja R, Hirsch IB, Naik RG, et al. Islet cell antibodies and glutamic acid decarboxylase antibodies, but not the clinical phenotype, help to identify type 1(1/2) diabetes in patients presenting with type 2 diabetes. Metabolism 2001; 50:1008-13.

25. Turner R, Stratton I, Horton V, et al. Autoantibodies to islet-cell cytoplasm and glutamic acid decarboxylase for prediction of insulin requirement in type 2 diabetes (UKPDS 25). Lancet 1997; 350:1288-93.

26. Borg H, Gottsäter A, Fernlund P, Sundkvist G. A 12-year prospective study of the relationship between islet antibodies and beta-cell function at and after the diagnosis in patients with adult-onset diabetes. Diabetes 2002; 51:1754-62.

27. Borg H, Gottsäter A, Landin-Olsson M, Fernlund P, Sundkvist G. High levels of antigen-specific islet antibodies predict future beta-cell failure in patients with onset of diabetes in adult age. J Clin Endocrinol Metab 2001; 86:3032-8

28. Kobayashi T, Nakanishi K, Murase T, Kosaka K. Small doses of subcutaneous insulin as a strategy for preventing slowly progressive beta-cell failure in islet cell antibody-positive patients with clinical features of NIDDM. Diabetes 1996; 45:622-6.

29. Anastasi E, Dotta F, Tiberti C, et al. Insulin prophylaxis down-regulates islet antigen expression and islet autoimmunity in the low-dose Stz mouse model of diabetes. Autoimmunity 1995; 29:249-56.

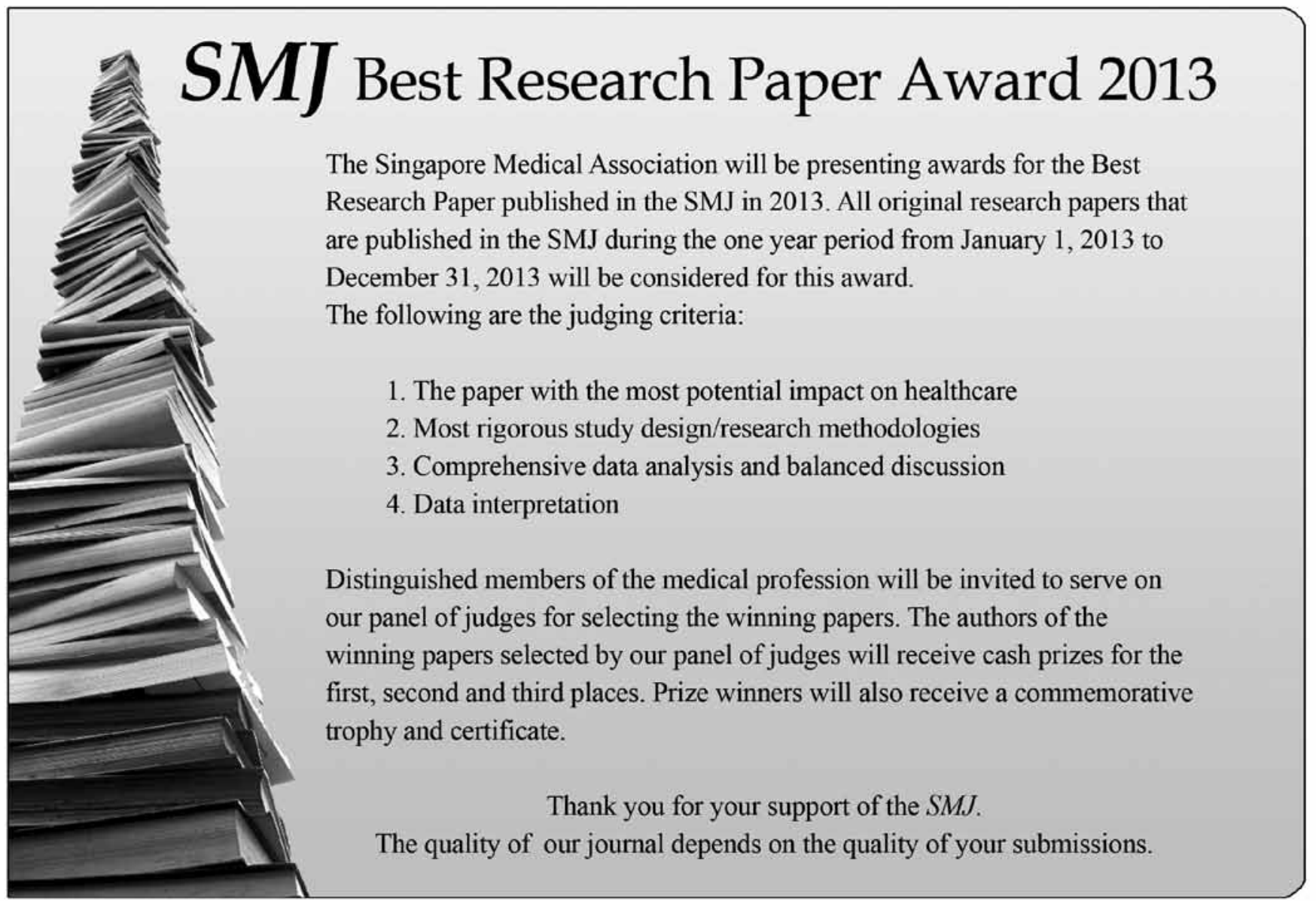

